The final publication is available at SAGE via

http://journals.sagepub.com/doi/abs/10.1177/1359105317718925 


\title{
Shame and emotion regulation in inflammatory bowel disease: \\ Effects on psychosocial functioning
}

\author{
Inês A. Trindade, MSc, PhD student $1 *$ \\ Cláudia Ferreira, $\mathrm{PhD}^{1}$ \\ José Pinto-Gouveia, $\mathrm{MD}, \mathrm{PhD}^{1}$
}
${ }^{1}$ CINEICC - Cognitive and Behavioural Centre for Research and Intervention Faculty of Psychology and Educational Sciences
University of Coimbra, Portugal

* Correspondence concerning this article should be addressed to:

Inês A. Trindade

Email: ines.almeidatrindade@gmail.com

CINEICC, Faculdade de Psicologia e Ciências da Educação,

Universidade de Coimbra

Rua do Colégio Novo, Apartado 6153

3001-802 Coimbra, Portugal

Telephone: (+351) 239851450

Fax: (+351) 239851462 


\title{
Shame and emotion regulation in inflammatory bowel disease: \\ Effects on psychosocial functioning
}

\begin{abstract}
Although patients with inflammatory bowel disease seem to be prone to high levels of shame, the mechanisms behind the impact of chronic illness-related shame on patients' functioning have not been explored yet. This study aims to address these gaps using a sample of 161 patients with inflammatory bowel disease who completed self-report measures on an online survey. The results from path analyses showed that chronic illnessrelated shame presented direct and indirect effects on psychological health $\left(R^{2}=.66\right)$ and social relationships $\left(R^{2}=.46\right)$. The indirect effects were mediated by experiential avoidance and uncommitted living. Possible explanations to these findings and clinical implications are discussed.
\end{abstract}

Keywords: chronic illness-related shame, emotion regulation, experiential avoidance, inflammatory bowel disease, uncommitted living 


\section{Introduction}

Inflammatory bowel disease (IBD) presents clinical characteristics and treatment implications that can have a profound impact on patients' psychological functioning and personal relationships (e.g. Graff et al., 2006). IBD encompasses two related illnesses, Crohn's disease (CD) and ulcerative colitis (UC), characterized by a chronic and relapsing inflammation within the gastrointestinal tract. Patients with IBD may experience severe symptoms such as abdominal pain, diarrhoea, rectal bleeding, faecal urgency and incontinence, weight loss and fatigue. Due to the relapsing nature of the disease, these symptoms have an unexpected and intermittent course, and their frequency and intensity vary between periods of exacerbations and relative inactivity. The presence of complications associated with IBD is also common as several patients report having fistulas, abscesses, arthritis and dermatological, ocular and gynaecological complaints (e.g. Levine and Burakoff, 2011). Life-threatening problems might emerge as bowel perforation, toxic megacolon and the development of colon cancer.

Some patients may be submitted to surgical procedures (which may comprise an ostomy) to manage more severe symptomatology (Crohn's and Colitis Foundation of America (CCFA), 2010). Nevertheless, treatment during exacerbations of the disease usually involves taking corticosteroids, known to cause side effects such as facial swelling and weight gain. Also, patients are typically encouraged to follow a certain diet (as high meat, alcohol, dairy and fibre intakes can aggravate symptomatology) and to eat small and frequent meals (e.g. CCFA, 2005; Jowett et al., 2014; MacDermott et al., 2015).

The features of IBD usually interfere with the patient's work, social and leisure activities due to symptomatology that may emerge unexpectedly. It is reported that patients sometimes feel the need to cancel planned activities due to fatigue, pain or fear of faecal incontinence, which may not be adequately understood by others (Casati and 
Toner, 2000). Furthermore, IBD symptomatology and its treatment may also decrease patients' body image and interfere with sexual functioning (Sainsbury and Heatly, 2005; Trachter et al., 2002); in fact, some patients completely avoid intercourse due to fears of incontinence, abdominal pain or dyspareunia (Moody et al., 1992; Moody and Mayberry, 1993).

Moreover, concerns about not reaching full potential, feeling a burden to others and being ostracized by society are also typically reported by patients, as well as fears about developing cancer or being infertile (Casati and Toner, 2000; Mountifield et al., 2009; Trachter et al., 2002). In this line, feelings of isolation, stigmatization and shame are common among patients with IBD (Casati and Toner, 2000; Hall et al., 2005), and their mental health and social relationships are generally impaired (e.g. Graff et al., 2006; Kurina et al., 2001; Rowlinson, 1999). For example, Walker et al. (2008) have reported that patients with IBD are at least twice as likely to develop a depressive disorder in comparison with normal controls with similar ages and backgrounds.

\section{Shame and chronic illness}

Humans are particularly focused and responsive to what others think about the self (Gilbert, 2002, 2007) and feel the desire to create positive images of themselves in the eyes of others (Gilbert, 1997; Gilbert and McGuire, 1998). This innate desire is associated with the fact that human social relationships have evolved around the need to appear attractive and to be chosen for important roles (e.g. ally, friend, lover; Gilbert, 2000), due to the substantial benefits of social acceptance and the detrimental consequences of social rejection (ultimately death; Baumeister and Leary, 1995).

Shame is a self-conscious emotion that can act as a warning of the risk of exclusion by the social group (Gilbert, 2007). Although this experience presents an evolutionary 
function, it is usually considered painful and involves the perception that one presents unfavourable and unattractive personal attributes (e.g. physical appearance, personality traits, an illness) or has displayed behaviours that others may find inappropriate and result in some form of criticism, attack or rejection (Gilbert, 1998; Tangney and Fischer, 1995). Shame thus leads to the perception that the self exists negatively in the mind of others as inferior, inadequate or flawed; these attributes can be further internalized leading to selfblame and self-criticism (Gilbert, 1998, 2007). Furthermore, high levels of shame may become pathological and are extensively and highly associated with lower quality of life (QoL) and increased psychopathology (e.g. Kim et al., 2011; Tangney and Dearing, 2002), including anxiety (Tangney et al., 1992) and depression (e.g. Cheung et al., 2004; Matos and Pinto-Gouveia, 2010). Chronic patients, especially those with illnesses involving intestinal symptomatology or visible symptomatology, are theoretically considered to be more disposed to experience shame feelings (e.g. Casati et al., 2000; Kellett and Gilbert, 2001). In IBD particularly, shame seems to be of especial relevance for patients' psychological functioning due to the features and consequences of the illness. Nevertheless, the study of this specific emotion is not yet fully developed, and its associated mechanisms have not been explored.

\section{Experiential avoidance and uncommitted living in chronic illness}

Recent research has pointed that more than the experience of adverse events (such as physical symptomatology, shame and related thoughts and emotions), it is the way the individual deals with those events that determine their impact on one's well-being (Segal et al., 2002). That is, the suffering that results from events perceived as painful seems to be associated with the emotion regulation strategies one uses to cope. One increasingly studied maladaptive emotion regulation process refers to experiential avoidance, a key 
theme of several contextual behavioural therapies, such as acceptance and commitment therapy (ACT; Hayes et al., 2012).

Experiential avoidance occurs when an individual is unwilling to remain in contact with particular private experiences (e.g., bodily sensations, emotions, thoughts, memories, or behavioral predispositions) and takes steps to alter the form or frequency of these events and the contexts that occasion them' (Hayes et al., 1996: 1154). This process thus involves attempts to control, limit or avoid internal events, even when it results in significant personal costs. Indeed, although experiential avoidance may have momentaneous positive outcomes, in the long-term it causes a paradoxical exacerbation of the internal events one is trying to avoid (Chawla and Ostafin, 2007; Ruiz, 2010). Furthermore, experiential avoidance strategies are usually inflexible, behaviourally restrictive and hardly meaningful. With time, these avoidance patterns become less and less sensitive to the opportunities offered by the context and restrain the individual to behave in accordance with important goals and lead a valued, fulfilled life (Hayes et al., 2006). The person thus starts behaving incoherently with the valued life he or she wants to follow (e.g. being healthy, being a good friend) to keep trying to control internal events (Hayes et al., 2006). For example, it is reported that adolescents with diabetes may intentionally miss insulin injections to avoid painful thoughts related to weight gain, which leads to long-term health problems (Hadlandsmyth et al., 2013). Furthermore, considerable evidences have demonstrated that these processes account for a considerable amount of the variance of several psychosocial outcomes, such as QoL, anxiety, depression or disease self-management (for a review, see Hayes et al., 2006). In a recent study with IBD patients, experiential avoidance has significantly mediated the associations between IBD symptomatology and physical and psychological functioning (Trindade, Ferreira, \& Pinto-Gouveia, 2015b). It seems that patients perceive disease- 
related sensations, thoughts or memories as threatening and try to control or avoid them, which only amplifies their impact on QoL. Conversely, acceptance of internal experiences has been associated with positive outcomes in several health settings such as chronic pain and irritable bowel syndrome (Ferreira, 2011; McCracken and Eccleston, 2006; McCracken and Vowles, 2008). Likewise, committed living (consistent with one's personal values) has been empirically linked to decreased neuroendocrine and psychological responses to stress, higher levels of life fulfilment and improved psychosocial indices (Cresswell et al., 2005; Trompetter et al., 2013; Wilson and Murrel, 2004). These data seem to highlight the need to further study experiential avoidance and uncommitted living in chronic conditions such as IBD.

\section{Aims}

The aim of this article was therefore to explore the role of these maladaptive processes in the psychosocial functioning of patients with IBD. We hypothesize that experiential avoidance and uncommitted living will significantly mediate the effect of chronic illness-related shame on psychological health and social relationships.

\section{Materials and Methods}

\section{Procedures}

This article is part of a larger research project which globally focuses on studying the role of emotion regulation processes in QoL of patients with IBD. This project was ethically approved by the Portuguese Association for IBD (APDI) which electronically invited its registered patients to participate in the research. Respondents were properly informed about the aims, procedures and confidentiality nature of the research, and those who agreed to participate gave their informed consent and completed an online survey. 
Participants who were pregnant or presented severe diseases other than IBD (e.g. breast cancer) or psychiatric disorders (e.g. anxiety disorders, depression, bipolar disorder) were later excluded from the research.

\section{Measures}

The research protocol included demographic and medical history questions. Medical information comprised form of IBD, time since IBD diagnosis, frequency of IBD symptomatology during the previous month (physical symptoms measured on a 6-point scale), presence of associated medical complications, number of hospital admissions and undergone surgeries and type of surgery (if any). Furthermore, study participants completed the Portuguese versions of a series of self-report instruments:

Chronic Illness-related Shame Scale. This seven-item scale measures shame specifically associated with a chronic illness and its symptomatology. Items (e.g. 'I feel that my illness is embarrassing', 'I feel inadequate because of my illness and symptoms') are rated on a 5-point Likert scale ranged from 0 (Never true) to 4 (Always true). The Chronic Illness-Related Shame Scale (CISS) has revealed very good psychometric properties $(\alpha=.91)$ in its original study, which was conducted with Portuguese chronic patients with age ranges similar to the present sample (Trindade, Ferreira, \& PintoGouveia, 2015a).

Acceptance and Action Questionnaire-II. This questionnaire presents 10 items on a scale with 7 points (from 1: Never true to 7: Always true) and assesses participants' level of experiential avoidance (e.g. 'I worry about not being able to control my worries and feelings'; Bond et al., 2011; Pinto-Gouveia et al., 2012). The Acceptance and Action 
Questionnaire-II (AAQ-II) has presented good internal consistencies in the original study (with a mean $\alpha$ of .84 across different six samples) in the Portuguese validation study ( $\alpha$ $=.90)$.

Engaged Living Scale-9. The ELS-9 (Trompetter et al., 2013; Trindade, Ferreira, Pinto-Gouveia, \& Nooren, 2015) assesses the process of engaged living, as conceptualized in ACT (Trompetter et al., 2013). Before responding to the scale, participants read a short introduction that clarifies the constructs of engaged living, valuing and the term 'values' (Strosahl et al., 2004), so the items are adequately understood by the respondent. Items are scored on a 5-point Likert scale (1: Completely disagree; 5: Completely agree) and include two factors, Valued Living (VL) and Life Fulfilment (LF). In this study, only the VL subscale was used (e.g. 'I believe that my values are really reflected in my behaviour'; 'My emotions don't hold me back from doing what's important to me') and scores were reversed to obtain a measure of uncommitted living. Furthermore, the nine-item version of the scale was used. This nineitem scale has revealed adequate psychometric properties $\left(\alpha_{\mathrm{total}}=.88 ; \alpha_{\mathrm{VL}}=.76 ; \alpha_{\mathrm{LF}}=\right.$ $.89)$.

World Health Organization Brief Quality of Life Assessment Scale. The World Health Organization Brief Quality of Life Assessment Scale (WHOQOL-BREF) is shortform scale of subjective QoL on four different domains: physical health, environmental health, psychological health and social relationships (Canavarro et al., 2009; WHOQOL Group, 1998). The scale presents 26 items rated on a 5-point Likert scale, with higher scores indicating higher levels of perceived QoL. The WHOQOL-BREF has presented adequate psychometric characteristics in the original ( $\alpha$ s between .66 and .84) and 
Portuguese validation studies ( $\alpha$ s between .67 and .87). In this study, only two domains were used, psychological health (e.g. 'How often do you have negative feelings such as blue mood, despair, anxiety, depression?') and social relationships ('How satisfied are you with your personal relationships?').

These instruments demonstrated adequate to excellent internal reliabilities (in accordance with Kline's (2000) recommendations) in this study (Table 1).

\section{Participants}

This study's sample comprised 161 patients with IBD (52 males and 109 females) with ages ranging between 19 and 76 years old $(M=36.73$; standard deviation $(S D)=$ 10.73). Their educational level ranged between seventh grade and $\mathrm{PhD}$ (completed years of education: $M=14.55 ; S D=2.71$ ). Furthermore, 26,50 and 5 per cent of the participants presented high, medium and low socio-economic status, respectively. The rest of the participants were college students $(11 \%)$, unemployed $(5 \%)$ or retired $(3 \%)$ individuals. Regarding marital status, 52 per cent of the participants were married or cohabitating, 40 per cent were single, 7 per cent divorced and 1 per cent widowed.

Concerning medical data, 89 participants $(55 \%)$ had been previously diagnosed with $\mathrm{CD}, 70$ with UC (44\%) and $2(1 \%)$ were undergoing the diagnostic process. Time since diagnosis varied between 1 year and 37 years and had a mean of 9.32 years $(\mathrm{SD}=$ 6.68 years). Furthermore, 31 (19\%) participants reported having active disease during the moment of assessment. The most reported symptoms were gas $(M=3.55 ; S D=1.38)$, fatigue $(M=3.19 ; S D=1.61)$, abdominal distension $(M=2.74 ; S D=1.77)$ and abdominal pain $(M=2.47 ; S D=1.43)$. Moreover, 48 participants $(30 \%)$ reported having one or more IBD associated medical complications such as osteoarticular pain, dermatological 
manifestations and ocular problems. Forty-five patients (28\%) had been submitted to at least one IBD-related surgery, of whom 7 (4\%) reported having a stoma; the number of undergone surgeries varied between 0 and $10(M=1.68 ; S D=1.57)$.

\section{Statistical analyses}

Descriptive and Pearson correlation analyses were conducted using the software SPSS (v. 22; IBM Corp, 2013) to explore the associations between study variables (Cohen et al., 2003). A path analysis tested a theoretical model that analysed whether experiential avoidance and uncommitted living would mediate the relationships of chronic illnessrelated shame with psychological health and social relationships. This analysis was performed with the Amos software (Arbuckle, 2006) using maximum likelihood as estimation method. Path analysis is a type of structural equation modelling (SEM) that analyses structural associations and the significance of direct and indirect paths (e.g. Schumacker and Lomax, 2004). This analysis was conducted using the bootstrap procedure (with 5000 samples) to create 95 per cent bias-corrected confidence intervals (CIs) around the standardized estimates of total, direct and indirect effects. If the interval between the lower and the upper bounds of the 95 per cent bias-corrected CI does not comprise zero, the effects are statistically significant (Kline, 2005). The adjustment of the tested model to the empirical data was examined recurring to the chi-square goodness-offit (which reveals a good fit when nonsignificant; Hair et al., 1998), the root mean squared error of approximation (RMSEA; which reveals a good adjustment when values are inferior to .06; Hu and Bentler, 1999) and the comparative fit index (CFI) and the Tucker and Lewis Index (TLI) which reveal a good model fit when values are superior to .95 (Hooper et al., 2008; Hu and Bentler, 1999). 


\section{Results}

\section{Correlations}

The results from the correlation analyses revealed that chronic illness-related shame was negatively associated with age (with a small magnitude) and psychological health and social relationships (with large magnitudes). Furthermore, chronic illnessrelated shame presented positive, moderate to high, relationships with IBD symptomatology and the processes of experiential avoidance and uncommitted living.

Moreover, experiential avoidance was positively correlated with IBD-associated medical complications, IBD symptomatology and uncommitted living and presented negative and large associations with psychological health and social relationships.

Uncommitted living also presented positive correlations with IBD symptomatology and was negatively linked to psychological health and social relationships. These two lastly referred variables were, in turn, highly correlated with each other and moderately associated with IBD symptomatology.

The impact of chronic illness-related shame, experiential avoidance and uncommitted living on IBD patients' psychological health and social relationships

Skewness and Kurtosis' values' analysis demonstrated that the variables did not present a considerable bias to normal distribution $(\mathrm{Sk}=|0.13-0.47| ; \mathrm{Ku}=|0.20-0.77|)$. The assumption of normality was confirmed through the visual inspection of the distributions (Kline, 2005).

The tested theoretical model (Figure 1) explored whether experiential avoidance and uncommitted living significantly mediate the effect of chronic illness-related shame 
on psychological health and social relationships (with IBD symptomatology, associated medical complications and age as covariates).

This model had 34 parameters and explained 42 per cent of experiential avoidance, 15 per cent of uncommitted living, 66 per cent of psychological health and 46 per cent of social relationships. Furthermore, all individual path coefficients were statistically significant, and the model presented an excellent adjustment to the empirical data, with a non-significant chisquare of $\chi_{(14)}^{2}=19.41, p=.150$, and the following goodness-of-fit indices: $\mathrm{CFI}=.99 ; \mathrm{TLI}=.98 ;$ and RMSEA $=.05, \mathrm{p}=.464, \mathrm{CI}$ from .00 to .10 .

Please insert Figure 1 around here

Chronic illness-related shame presented direct effects of .65 on experiential avoidance $\left(b_{\mathrm{CISS}}=1.08\right.$; standard error $\left.(\mathrm{SE})=.10 ; Z=10.61 ; p<.001\right), .39$ on uncommitted living $\left(b_{\mathrm{CISS}}=.19 ; \mathrm{SE}=.04 ; Z=-5.25 ; p<.001\right),-.22$ on psychological health $\left(b_{\text {CISS }}=-.65 ; \mathrm{SE}=.18 ; Z=-3.62 ; p<.001\right)$ and -.21 on social relationships $\left(b_{\text {CISS }}\right.$ $=-.73 ; \mathrm{SE}=.27 ; Z=-2.72 ; p<.001)$.

Psychological health and social relationships were also directly predicted by experiential avoidance with magnitudes of $-.55\left(b_{\mathrm{AAQ}-7}=-.93 ; \mathrm{SE}=.11 ; Z=-8.68 ; p<\right.$ $.001)$ and $-.33\left(b_{\mathrm{AAQ}-7}=-.69 ; \mathrm{SE}=.17 ; Z=-4.09 ; p<.001\right)$, respectively, and by uncommitted living $\left(\beta=-.18 ; b_{\mathrm{ELS}}=-1.08 ; \mathrm{SE}=.32 ; Z=3.34 ; p<.001 ; \beta=-.28 ; b_{\mathrm{ELS}}\right.$ $=-2.07 ; \mathrm{SE}=.49 ; Z=4.24 ; p<.001$, respectively).

Furthermore, these emotion regulation processes (experiential avoidance and uncommitted living) significantly mediated the association between chronic illnessrelated shame and psychological health with a combined indirect effect of $-.42(95 \% \mathrm{CI}$ 
$=-1.58$ to $-.92 ; p<.001)$. The specific indirect effect of experiential avoidance was -.35 , while uncommitted living presented a specific indirect effect of -.07 (Hayes et al., 2011). The association between chronic illness-related shame and psychological health thus presented a total effect of -.64 .

Likewise, the relationship between chronic illness-related shame and social relationships was also significantly mediated by the mechanisms of experiential avoidance and uncommitted living, presenting a combined indirect effect of -.32 (95\% $\mathrm{CI}=-1.55$ to $-.71 ; \mathrm{p}<.001)$ and a total effect of -.53 . In this association, the specific contributions of experiential avoidance and uncommitted living were -.21 and -.11 , respectively (Hayes et al., 2011).

\section{Discussion}

Recent literature has highlighted the importance to further analyse the effect of experiential avoidance and uncommitted living on chronic conditions such as IBD. The objective of this study was therefore to examine the mediator role of these emotion regulation processes on the effect of chronic illness-related shame on psychological health and social relationships, using a sample of 161 patients with IBD.

Concerning the conducted correlation analyses, it was interesting to note that chronic illness-related shame presented negative associations with age, IBD symptomatology and the studied emotion regulation processes, experiential avoidance and uncommitted living. These processes were, in turn, linked to each other and to greater IBD symptomatology, and in the case of experiential avoidance, to higher incidence of associated medical complications. The studied outcomes, psychological health and social relationships, showed positive moderate to high relationships with all studied variables except for age and associated medical complications. 
Regarding the main aim of the study, a model conducted through path analyses demonstrated that chronic illness-related shame presented direct and indirect effects on psychological health and social relationships. The indirect effects were larger $(-.42$ and -.32 , respectively) than the direct ones ( -.22 and -.21 , respectively) and were mediated by experiential avoidance and uncommitted living. Furthermore, this model accounted for 66 per cent of psychological health and 46 per cent of social relationships' variance and revealed an excellent fit to the empirical data. These findings thus suggest that chronic illness-related shame holds a detrimental impact on psychosocial functioning of patients with IBD; this impact might be explained by the features of the diseases. Indeed, symptomatology such as frequent and urgent diarrhoea, gas or incontinence may be a source of shame, possibly leading to feelings of inadequacy, inferiority, unattractiveness and isolation. Fears of not reaching full potential, being a burden to others or changes in body image (due to weight loss, corticosteroids intake or surgery) may also explain shame in patients with IBD. We may hypothesize that disease-related shame held a direct effect on patients' mental health and social relationships due to the perceived intensity of these feelings.

Nevertheless, this study's findings also suggest that the impact of shame feelings related to chronic illness seems to be amplified by the engagement in strategies aiming to control or avoid internal experiences and by the associated experience of living an uncommitted, unfulfilled life. In fact, these findings seem to corroborate that patients with IBD experience illness-related internal events (such as shame and related cognitions) as threatening and tend to engage in control and avoidance strategies. Also, our findings go in line with previous studies, by showing that this process leads to more suffering in the long term (e.g. Hayes et al., 2006; Ruiz, 2010) and is usually associated with a narrow 
behavioural repertoire, inconsistent with important personal values (e.g. Hayes et al., 2012).

These findings suggest that patients with IBD may react to disease-related shame by trying to control or avoid unwanted internal experiences, which may gradually lead to rigid patterns of behaviour. For instance, patients feeling shame associated with physical appearance or diet changes, frequency of toilet use or fears of incontinence may deal with these feelings by trying to control them through the avoidance of work or social contexts. Also, patients may deal with shame related to symptomatology, fears of developing cancer or having difficulty in performing daily activities (e.g. due to fatigue) by avoiding discussing these issues with colleagues, friends or intimate partners, decreasing the proximity and quality of their relationships with others. Overall, these avoidance patterns may compromise important areas of the patient's life, inhibiting the engagement in actual valued activities. It can thus be suggested that the mechanisms of experiential avoidance and uncommitted living may intensify psychological suffering of patients with IBD, by amplifying the pernicious effect of chronic illness-related shame on psychological health and social relationships.

While interpreting these findings, it is important to take into consideration a few limitations. First, we acknowledge that the cross-sectional nature of the study may be a main limitation to the study and that future investigations should test the analysed model using longitudinal designs. Given this is the first study to explore the mechanisms underlying chronic illness-related shame, it would be important to further study other emotion regulation processes that might be involved (e.g. chronic illness-related experiential avoidance and cognitive fusion; fear of compassion). Furthermore, the type of data collection (through an Internet survey using self-report questionnaires) and the limited sample size might have compromised the collection of a representative sample of 
the Portuguese IBD population and the presentation of definite results; in this way, future studies should be performed with larger samples of patients recruited in medical settings, using clinical interviews. Finally, the tested model should also be analysed in patients of other cultures and countries.

Nevertheless, we believe this study significantly contributes to the comprehension of psychosocial impairment among patients with IBD, by demonstrating the significant role of emotion regulation processes on the impact of chronic illness-related shame on psychological health and social relationships. In this line, it seems that more emphasis to the psychological aspects of IBD should be given in treatment programmes. The present findings indeed suggest that patients with IBD would benefit from compassionate and acceptance-based psychotherapies, to develop more adaptive ways of dealing with illness-related experiences.

\section{Funding}

Research by the first author (Inês A. Trindade) is supported by a Ph.D. Grant (SFRH/BD/101906/2014) sponsored by FCT (Portuguese Foundation for Science and Technology).

\section{Acknowledgements}

We are grateful for the contribution given by the Portuguese Association for IBD (Associação Portuguesa para a Doença Inflamatória do Intestino; APDI) during the recruitment process of the current research project.

\section{References}

Arbuckle JL (2006) Amos (Version 7.0) [Computer Program]. Chicago, IL: SPSS. 
Baumeister RF and Leary MR (1995) The need to belong: Desire for interpersonal attachments as a fundamental human motivation. Psychological Bulletin 117: $497-529$.

Bond FW, Hayes SC, Baer RA, et al. (2011) Preliminary psychometric properties of the Acceptance and Action Questionnaire-II: A revised measure of psychological inflexibility and experiential avoidance. Behavior Therapy 42: 676-688.

Canavarro MC, Serra AV, Simoes MR, et al. (2009) Development and psychometric properties of the World Health Organization Quality of Life Assessment Instrument (WHOQOL-100) in Portugal. International Journal of Behavioral Medicine 16(2): 116-124.

Casati J, Toner BB, de Rooy EC, et al. (2000) Concerns of patients with inflammatory bowel disease: A review of emerging themes. Digestive Diseases and Sciences 45: $26-31$.

Chawla N and Ostafin B (2007) Experiential avoidance as a functional dimensional approach to psychopathology: An empirical review. Journal of Clinical Psychology. Epub ahead of print 2 August. DOI: 10.1002/jclp.20400.

Cheung MSP, Gilbert P and Irons C (2004) Na exploration of shame, social rank and rumination in relation to depression. Personality and Individual Differences 36: $1143-1153$.

Cohen J, Cohen P, West S, et al. (2003) Applied Multiple Regression/Correlation Analysis for the Behavioral Sciences. (3rd edn). Mahwah, NJ: Lawrence Erlbaum Associates.

Cresswell JD, Welch WT, Taylor SE, et al. (2005) Affirmation of personal values buffers neuroendocrine and psychological stress response. Psychological Science 16: 846-851. 
Crohn's and Colitis Foundation of America (CCFA) (2005) Living with IBD

[Brochure]. New York: Crohn's and Colitis Foundation of America.

Crohn's and Colitis Foundation of America (CCFA) (2010) Surgery for Crohn's

Disease and Ulcerative Colitis [Brochure]. New York: Crohn's and Colitis Foundation of America.

Ferreira NB (2011) Investigating the role of psychological flexibility and the use of an acceptance and commitment therapy based intervention in irritable bowel syndrome. Doctoral dissertation, University of Edinburgh, Edinburgh.

Gilbert P (1997) The evolution of social attractiveness and its role in shame, humiliation, guilt and therapy. British Journal of Medical Psychology 70: 113147.

Gilbert P (1998) What is shame? Some core issues and controversies. In: Andrews B and Gilbert P (eds) Shame: Interpersonal Behavior, Psychopathology, and Culture. New York: Oxford University Press, pp. 3-31.

Gilbert P (2000) The relationship of shame, social anxiety and depression: The role of the evaluation of social rank. Clinical Psychology \& Psychotherapy 7(3): 174189.

Gilbert P (2002) Body shame: A biopsychosocial conceptualization and overview with treatment implications. In: Gilbert P and Miles J (eds) Body Shame: Conceptualization, Research and Treatment. New York: Routledge, pp. 3-47. Gilbert P (2007) The evolution of shame as a marker for relationship security. In: Tracy JL, Robins RW and Tangney JP (eds) The Self-Conscious Emotions: Theory and Research. New York: Guilford Press, pp. 283-309. 
Gilbert P and McGuire MT (1998) Shame, status, and social roles: Psychobiology and evolution. In: Gilbert P and Andrews B (eds) Shame: Interpersonal Behavior, Psychopathology, and Culture. New York: Oxford University Press, pp. 99-125.

Graff LA, Walker JR, Lix L, et al. (2006) The relationship of inflammatory bowel disease type and activity to psychological functioning and quality of life. Clinical Gastroenterology and Hepatology 4(12): 1491-1501.

Hadlandsmyth K, White KS, Nesin AE, et al. (2013) Proposing an acceptance and commitment therapy intervention to promote improved diabetes management in adolescents: A treatment conceptualization. International Journal of Behavioral Consultation and Therapy 7(4): 12-15.

Hair JF, Anderson RE, Tatham RL, et al. (1998) Multivariate Data Analysis. Upper Saddle River, NJ: Prentice-Hall International.

Hall NJ, Rubin GP, Dougall A, Hungin PS, and Neely J (2005) The fight for "healthrelated normality": A qualitative study of the experiences of individuals living with established inflammatory bowel disease (ibd). Journal of Health Psychology, 10(3):443-455. DOI: 10.1177/1359105305051433.

Hayes AF, Preacher KJ and Myers TA (2011) Mediation and the estimation of indirect effects in political communication research. In: Erik P and Holbert LR (eds) Sourcebook for Political Communication Research: Methods, Measures, and Analytical Techniques. Abingdon, UK: Taylor \& Francis, pp. 457-488.

Hayes SC, Luoma JB, Bond F, et al. (2006) Acceptance and commitment therapy: Model, processes and outcomes. Behaviour Research and Therapy 44: 1-25. Hayes SC, Strosahl KD, and Wilson KG (2012) Acceptance and commitment therapy: The process and practice of mindful change (2nd edn). New York: The Guilford Press. 
Hayes SC, Wilson KW, Gifford EV, Follette VM and Strosahl K (1996) Experiential avoidance and behavioral disorders: A functional dimensional approach to diagnosis and treatment. Journal of Consulting and Clinical Psychology, 64(6): 1152-1168. DOI: 10.1037/0022-006X.64.6.1152.

Hooper D, Coughlan J and Mullen MR (2008) Structural equation modelling: Guidelines for determining model fit. The Electronic Journal of Business Research Methods 6(1): 53-60.

Hu L and Bentler PM (1999) Cutoff criteria for fit indexes in covariance structure analysis: Conventional criteria versus new alternatives. Structural Equation Modeling: A Multidisciplinary Journal 6(1): 1-55.

IBM Corp Released (2013) IBM SPSS Statistics for Windows, Version 22.0. Armonk, NY: IBM Corporation.

Jowett SL, Seal CJ, Pearce MS, et al. (2004) Influence of dietary factors on the clinical course of ulcerative colitis: A prospective cohort study. Gut 53(10): 1479-1484.

Kellett S and Gilbert P (2001) Acne: A biopsychosocial and evolutionary perspective with a focus on shame. British Journal of Health Psychology 6: 1-24.

Kim S, Thibodeau R and Jorgensen R (2011) Shame, guilt, and depressive symptoms: A meta-analytic review. Psychological Bulletin 137(1): 68-96.

Kline P (2000) The Handbook of Psychological Testing (2nd edn). London: Routledge. Kline R (2005) Principles and Practice of Structural Equation Modeling (2nd edn). New York: The Guilford Press.

Kurina LM, Goldacre MJ, Yeates D, et al. (2001) Depression and anxiety in people with inflammatory bowel disease. Journal of Epidemiology and Community Health 55(10): 716-720. Levine JS and Burakoff R (2011) Extraintestinal manifestations 
of inflammatory bowel disease. Journal of Gastroenterology and Hepatology $7: 235-241$.

McCracken L and Vowles KE (2008) A prospective analysis of acceptance of pain and values-based action in patients with chronic pain. Health Psychology 27(2): 215220.

McCracken LM and Eccleston C (2006) A comparison of the relative utility of coping and acceptance-based measures in a sample of chronic pain sufferers. European Journal of Pain 10(1): 23-29.

McDermott E, Mullen G, Moloney J, et al. (2015) Body image dissatisfaction: Clinical features, and psychosocial disability in inflammatory bowel disease. Inflammatory Bowel Diseases 21(2): 353-360.

Matos M and Pinto-Gouveia J (2010) Shame as a traumatic memory. Clinical Psychology and Psychotherapy 17(4): 299-312.

Moody G, Probert CSJ, Srivastava EM, et al. (1992) Sexual dysfunction amongst women with Crohn's disease: A hidden problem. Digestion 52(3-4): 179-183. Moody GA and Mayberry JF (1993) Perceived sexual dysfunction amongst patients with inflammatory bowel disease. Digestion 54(4): 256-260.

Mountifield R, Bampton P, Prosser R, et al. (2009) Fear and fertility in inflammatory bowel disease: A mismatch of perception and reality affects family planning decisions. Inflammatory Bowel Diseases 15(5): 720-725.

Pinto-Gouveia J, Gregorio S, Dinis A, et al. (2012) Experiential avoidance in clinical and nonclinical samples. International Journal of Psychology and Psychological Therapy 12(2): 139-156.

Rowlinson A (1999) Inflammatory bowel disease: Importance of partnership in care. British Journal of Nursing 8(15): 1013-1028. 
Ruiz FJ (2010) A review of acceptance and commitment therapy (ACT) empirical evidence: Correlational, experimental psychopathology, component and outcome studies. International Journal of Psychology and Psychological Therapy 10(1): $125-162$.

Sainsbury A and Heatley RV (2005) Review article: Psychosocial factors in the quality of life of patients with inflammatory bowel disease. Alimentary Pharmacology \& Therapeutics 21(5): 499-508.

Schumacker RE and Lomax RG (2004) A Beginner's Guide to Structural Equation Modeling (2nd edn). Mahwah, NJ: Lawrence Erlbaum Associates.

Segal ZV, Williams JMG and Teasdale JD (2002) Mindfulness-Based Cognitive Therapy for Depression: A New Approach to Preventing Relapse. New York: Guilford Press.

Strosahl K, Hayes SC and Wilson KG (2004) Na acceptance and commitment therapy primer: Core therapy processes, intervention strategies, and therapist competencies. In: Hayes SC and Strosahl K (eds) A Practical Guide to Acceptance and Commitment Therapy. New York: Springer, pp. 31-58.

Tangney J and Fischer K (1995) Self-Conscious Emotions: The Psychology of Shame, Guilt, Embarrassment, and Pride. New York: Guilford Press.

Tangney JP and Dearing RL (2002) Shame and Guilt. New York: Guilford Press. Tangney JP, Wagner P and Gramzow R (1992) Proneness to shame, proneness to guilt, and psychopathology. Journal of Abnormal Psychology 101: 469-478.

The WHOQOL Group (1998) The World Health Organization Quality of Life Assessment (WHOQOL): Development and general psychometric properties. Social Science \& Medicine 46: 1569-1585. 
Trindade IA, Ferreira C and Pinto-Gouveia J. (2015a) Chronic illness-related shame: Development of a new scale and novel approach for IBD patients' depressive symptomatology. Submitted manuscript.

Trindade IA, Ferreira C and Pinto-Gouveia J (2015b) Inflammatory bowel disease: The harmful mechanism of experiential avoidance for patients' quality of life. Journal of Health Psychology 1-11. DOI: 10.1177/1359105315587142.

Trindade IA, Ferreira C, Pinto-Gouveia J, and Nooren L (2015) Clarity of personal values and committed action: Development of a shorter Engaged Living Scale. Journal of Psychopathology and Behavior Assesment. DOI: 10.1007/s10862-0159509-7.

Trompetter HR, ten Klooster PM, Schreurs KMG, Fledderus M, Westerhof GJ and Bohlmeijer ET (2013) Measuring values and committed action with the Engaged Living Scale (ELS): Psychometric evaluation in a nonclinical and chronic pain sample. Psychological Assessment 25(4): 1235-1246. DOI: 10.1037/a0033813.

Trachter AB, Rogers AI and Leiblum SR (2002) Inflammatory bowel disease in women: Impact on relationship and sexual health. Inflammatory Bowel Diseases 8(6): 413-421.

Trompetter HR, ten Klooster PM, Schreurs KMG, et al. (2013) Measuring values and committed action with the Engaged Living Scale (ELS): Psychometric evaluation in a nonclinical and chronic pain sample. Psychological Assessment 25(4): 12351246.

Walker JR, Ediger JP, Graff LA, et al. (2008) The Manitoba IBD cohort study: A population-based study of the prevalence of lifetime and 12-month anxiety and mood disorders. American Journal of Gastroenterology 103(8): 1989-1997. 
Wilson KG and Murrel AR (2004) Values work in acceptance and commitment therapy: Setting a course for behavioral treatment. In: Hayes SC, Follette VM and Linehan MM (eds) Mindfulness and Acceptance: Expanding the Cognitive-Behavioral Tradition. New York: Guilford Press, pp. 120-151. 


\section{Table 1}

Means (M), Standard Deviations (SD), Cronbach's alphas $(\alpha)$ and intercorrelation scores of the study variables $(N=161)$

\begin{tabular}{|c|c|c|c|c|c|c|c|c|c|c|}
\hline & $M$ & $S D$ & $\alpha$ & 1 & 2 & 3 & 4 & 5 & 6 & 7 \\
\hline 1. Age & 36.73 & 10.93 & - & 1 & & & & & & \\
\hline 2. IBD symptoms & 24.97 & 12.46 & - & .04 & 1 & & & & & \\
\hline 3. Associated complications & - & - & - & .10 & $.19 *$ & 1 & & & & \\
\hline 4. Chronic illness-related shame & 8.64 & 6.27 & .91 & $-.22 * *$ & $.41 * * *$ & $.20^{*}$ & 1 & & & \\
\hline 5. Experiential avoidance & 22.12 & 10.49 & .95 & -.08 & $.30 * * *$ & $.16^{*}$ & $.64 * * *$ & 1 & & \\
\hline 6. Uncommitted living & 10.95 & 3.02 & .78 & -.13 & $.21 * *$ & -.08 & $.38^{* * *}$ & $.48^{* * *}$ & 1 & \\
\hline 7. Psychological health & 63.98 & 18.47 & .87 & .07 & $-.40 * * *$ & -.15 & $-.64 * * *$ & $.78^{* * *}$ & $-.52 * * *$ & 1 \\
\hline 8. Social relationships & 61.96 & 22.00 & .81 & .01 & $-.33 * * *$ & -.12 & $-.53 * * *$ & $-.60 * * *$ & $-.52 * * *$ & $.75 * * *$ \\
\hline
\end{tabular}

Note: ${ }^{*} p<0.05 ;{ }^{* *} p<0.01 ;{ }^{* * *} p<0.001$.

Associated complications $=$ presence of IBD-related medical complications; Chronic Illness-related Shame $=$ CISS; Experiential avoidance $=$ AAQ-7;

Uncommitted living = VL subscale of the ELS-9 (with reversed scores); Psychological Health, Social Relationships = subscales of the WHOQOL-BREF. 


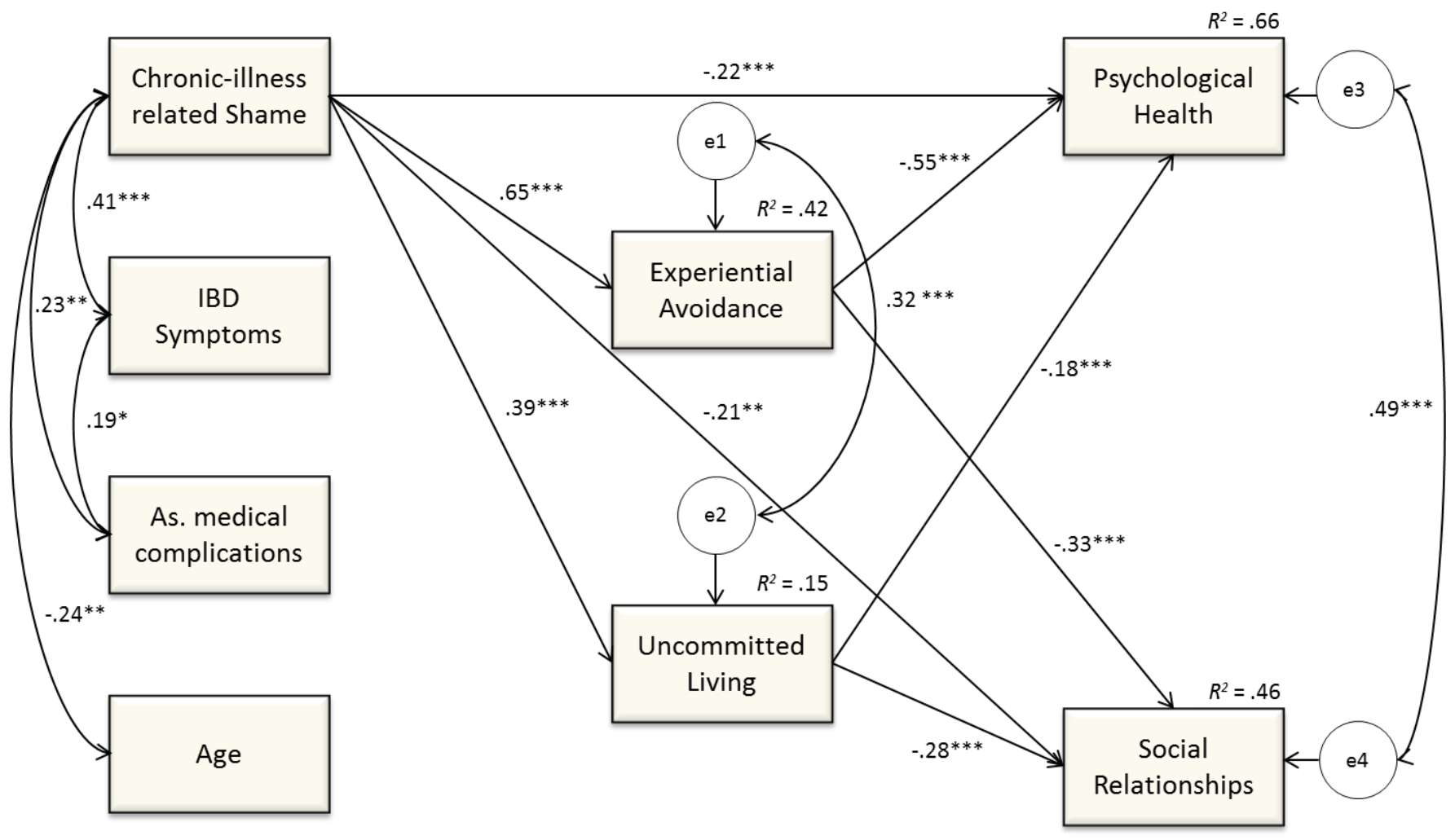

Note. Standardized path coefficients among variables are presented.

${ }^{*} p<0.05 ;{ }^{* *} p<0.01 ;{ }^{* * *} p<0.001$.

Associated complications $=$ presence of IBD-related medical complications; Chronic Illnessrelated Shame $=$ CISS $;$ Experiential avoidance $=$ AAQ-7; Uncommitted living $=$ VL subscale of the ELS-9 (with reversed scores); Psychological Health, Social Relationships = subscales of the WHOQOL-BREF. 